\section{SCALE: A FORTRAN program for reliability analysis on scales formed by linear combinations of variables}

\section{RAY W. COOKSEY \\ Colorado State University, Fort Collins, Colorado 80523}

SCALE is a program designed to assess the internal consistency reliabilities of a set of scales formed by either weighted or unweighted linear combinations of variables. This type of analysis is useful in understanding the nature and quality of scales derived from procedures such as factor analysis or cluster analysis. Nunnally (1967) has shown that scale reliability is an important factor in assessing how well the linearly combined variables reflect some common theme or "domain" of behavior. For this reason, Cronbach's (1951) coefficient of internal consistency (alpha) is computed by SCALE for each scale formed. The program is also designed to report several statistics relevant to the component variables of each scale and to compute an intercorrelation matrix between all scales formed. This correlation matrix contains both the raw (uncorrected) correlations between any two scales and the corrected (for unreliability) correlations. The program is constructed to deal with Likert-type scales with virtually any range of scale values.

Input. Input to the program consists of (1) a set of run parameters that govern various program options and the data-reading format, (2) scale information such as the number of variables per scale, scale labels (if desired), and component variable numbers, (3) weighting information (if desired) where weights such as regression weights are input by the user to be applied to their respective variables, and (4) the raw data that can be input on

This program was developed at Colorado State University with funding provided by the Rocky Mountain Forest and Range Experiment Station, USDA, Forest Service. Comments on an earlier draft by T. L. Dickinson, R. J. Loomis, P. A. Bell, and B. L. Driver are appreciated. cards, disk, or tape. If the weighting option is not desired, the program automatically assigns a unit weight to each variable in the analysis in order to provide unweighted linear combinations.

Output. Output includes (1) a list of selected program options, (2) a list of the scales, their labels (if any), and their component variable numbers (as a check for input accuracy), (3) component variable summary statistics (sums, means, and standard deviations) listed by scale membership, (4) summary statistics (sums, means, standard deviations, and Cronbach alpha reliability) for each scale, and (5) the intercorrelation matrix between all the scales formed in the analysis. Program SCALE also has the capability to deal with missing observations on variables and, for all summary statistics, the number of nonmissing cases is reported.

Computer and Language. The program is written (and documented) in ANSI FORTRAN IV and has been run on the CDC 6400 computer under the SCOPE 3.3 operating system; it is also functional on the CYBER 172 computer under the NOS 1.1 operating system.

Restrictions. The program can deal with up to 150 variables and can form and analyze up to 40 scales, with a limit of 20 variables in any one scale, in a given run. The core memory requirement for this program is $45 \mathrm{~K}$ on the CDC 6400 computer. The program executes in an average range of 10 to 20 computer sec.

Availability. A source listing, write-up, and simulated output can be obtained free of charge by writing Ray W. Cooksey, Department of Psychology, Colorado State University, Fort Collins, Colorado 80523.

\section{REFERENCES}

Cronbach, L. J. Coefficient alpha and the internal structure of tests. Psychometrika, 1951, 16, 297-334.

Nunnally, J. C. Psychometric theory. New York: McGrawHill, 1967.

(Accepted for publication June 20, 1978.) 